\title{
A Rare Case of Pelvic Primitive Neuroectodermal Tumor in a 37-Year-Old Patient
}

\author{
Florence Lai Tiong
}

\begin{abstract}
Primitive neuroectodermal tumors (PNETs) belong to the group of malignant bone tumors. Their frequency is about $1 \%$ of sarcoma. The principal distinctive feature is a translocation concerning the EWS gene or $\mathrm{t}(11 ; 22)(\mathrm{q} 24 ; \mathrm{q} 12)$. They are even rarer when located in the extra-skeletal pelvic region. We report a case of a 37 -year-old female patient with a PNET in the pelvic region that is found after a spontaneous abortion. The tumor is localized on the right latero-uterine ligament. The tumoral size is of $10 \mathrm{~cm}$. After the coelioscopic diagnosis, the patient was treated with neo-adjuvant chemotherapy according to vincristine, ifosfamide, doxorubicin, and etoposide (VIDE) protocol, which ensured an important diminishing of the size of the tumor. She then had a total radical hysterectomy associated with a lymph node resection. The diagnosis was confirmed by histology, immunohistology examinations and molecular biology by hybridization in situ. The tumoral cells were classically small and round. They expressed CD99 and vimentin. Translocation $\mathrm{t}(11 ; 12)(\mathrm{q} 24 ; \mathrm{q} 12)$ was positive. Two months later, the patient presented a tumoral relapse and received an adjuvant chemotherapy by vincristine, dactinomycin, and ifosfamide (VAI) twice with a limited chemical and hematological tolerance that brought along palliative treatment. Extra-skeletal PNETs found in the pelvic region are very rare. Our present case is also rare by the way in which it was found, by the age of the patient and its being in the pelvic region. Prognosis with these tumors is still very bad regardless of the optimization of a multidisciplinary answer. The research of new biomarkers and target therapy could be a hope for the treatment of these rare and pejorative tumors.
\end{abstract}

Keywords: Primitive; Neuroectodermal; Rare gynecologic tumor

\section{Introduction}

Primitive neuroectodermal tumors (PNETs) are rare [1] and

Manuscript accepted for publication November 19, 2015

Department of Oncology, Institut de Cancerologie du Gard, Rue du Professeur Henri Pujol, 30029 Nimes, France. Email: florencelt@hotmail.fr

doi: http://dx.doi.org/10.14740/jmc2372w aggressive tumors which are usually found in children and teenagers. Males are likely to get it but the rate of survival is higher in females. They are more frequently found in Caucasian people than Afro-Americans. Of the patients, $20-25 \%$ are metastatic when they are diagnosed and one-third will have a tumoral relapse within 5 years of diagnosis regardless of adequate treatment [2].

PNETs show a similar histology, immunohistochemistry, and cytogenetics as Ewing's sarcomas [3].

Patients with these tumors have been treated according to protocols designed for rhabdomyosarcomas using vincristine, doxorubicin, and cyclophosphamide with ifosfamide and etoposide [3].

In the literature, the pelvic localization is very rare; 35 cases have been reported by Burgers et al [4].

In this report, we describe a rare case of a pelvis PNET.

\section{Case Report}

A 37-year-old woman was sent in by her gynecologist for an asymptomatic abdominal mass on the right side of the uterus randomly discovered after a non-progressive pregnancy of 8 weeks. Her medical history consisted of a postpartum phlebitis and a miscarriage (G5P3 patient). An abdomino-pelvic scan showed the uterus and bladder pushed horizontally to the right by a heterogeneous mass presenting both liquid and solid appearance with arch-like calcifications. The tumoral size is of 10 $\mathrm{cm}$. The pelvic magnetic resonance imaging (MRI) showed an egg-like shape taking up the right side of the pelvis, with polycyclic outlines and a very heterogeneous matrix. There was no modification with a gadolinium injection. A coelioscopy was performed to take histological material. The pathological diagnosis of PNETs was made based on the presence of a tumoral proliferation composed of small round or spindle-shaped cells with a small amount of cytoplasma. We observed also a fibrovascular tumoral stroma and any tumoral necrosis. The immunohistochemical (all antibodies and revelation system were those of Dako ${ }^{\circledR}$ ) cell phenotype was positive for CD99 and vimentin and negative for CD56, chromogranin, synaptophysin, cytokeratin (AE1/AE3), epithelial membrane antigen, protein S100, desmin, CD3, CD20, estrogenic or progesterone receptors and alpha inhibin. The proliferation marker Ki-67 was very high at $80 \%$. 
Molecular biology using fluorescence in situ hybridization was positive for translocation $\mathrm{t}(11 ; 12)(\mathrm{q} 24 ; \mathrm{q} 12)$ (EWSR1 22q12 break $\left.\left(\operatorname{Kreatech}^{\circledR}\right)\right)$.

Any metastasis was revealed by positron emission tomography (PET) or classical radiology (chest X-ray).

Biologically, the liver and renal functions were normal and NSE was elevated $(96.3 \mathrm{ng} / \mathrm{mL})$.

The patient was treated by a neo-adjuvant chemotherapy with vincristine, ifosfamide, doxorubicin, and etoposide (VIDE) protocol for six cycles. After two and four cycles, the PET scan showed a considerable reduction in size $(4.8 \mathrm{~cm})$ and in metabolic intensity of the tumoral mass. We saw stability after six cycles. The patient then had surgery (increased colpohysterectomy but incomplete resection due to the mass infiltrating the near totality of the psoas muscle). The tumor continued growing. The patient became symptomatic 2 months later with diffuse abdominal pains. Two adjuvant chemotherapeutic treatments by vincristine, dactinomycin, and ifosfamide (VAI) were then undertaken with worse clinical and hematological tolerances. After palliative care, the patient died 1 month later.

\section{Discussion}

PNETs are a group of rare tumors. Their annual incidence is 2.9 per million population [5].

Stout first described PNET in 1918 [6].

Their understanding has significantly advanced over the last 25 years. They have a wide distribution of primary tumors sites, related to their supposed neural origin [7].

On microscopy, they appear as a monotonous collection of small round, darkly stained cells leading to the differential diagnosis with a rhabdomyosarcoma and a neuroblastoma. Fortunately, PNETs are characterized by a certain number of specific translocations of the EWS gene which is found on the chromosome 22 classifying these tumors in the PNET family/ Ewing sarcoma. Over added molecular anomalies often occur during the progression of the illness such as the p53 or the p16 mutations [8].

Because of their large size, their numerous distant metastases, their poor radiosensitivity and their incomplete surgical resection, pelvic PNETs often show a pejorative prognosis. Burgers et al described 35 cases ( 15 boys and 20 girls aged between 3 and 31 years). Twenty-four of these patients showed any metastasis in time of the primary diagnosis. All were treated by chemotherapy associated for 31 with radiotherapy. Moreover, four patients had surgery. Five patients developed metastasis (pulmonary and skeletal). The pulmonary metastases were operated on followed by chemotherapy and sometimes radiotherapy. Patients treated only by radiotherapy showed intrathoracic relapses. Skeletal metastases were developed in cases treated by radiotherapy first and chemotherapy.

The difference of the 5-year survival without progression between patients without and with metastasis was $19 \%$ and $40 \%$, respectively [4].

Polychemotherapy and surgery were the basis of the treat- ment of PNETs. Polychemotherapy uses five drug approaches: vincristine, ifosfamide, cyclophosphamide, etoposide and doxorubicin. Complete surgical excision reduces drastically the risk of local relapse but does not prevent metastatic spread [9].

\section{Conclusion}

Survival of patients with PNETs on a single location has considerably increased with the arrival of modern chemotherapies as the target therapy but also with new surgical techniques by coelioscopy. Prognosis for patients who already have a diffuse illness or who have no response of the initial treatment remains worse.

A better comprehension of signals may be a future path in the treatment of these rare tumors.

\section{References}

1. Simoens WA, Degryse HR. Primitive Neuroectodermal Tumors and Related Lesions. In: Md AMDS, Md PMP, Md LDB, Md FV, editors. Imaging of Soft Tissue Tumors [Internet]. Springer Berlin Heidelberg; 2001 [cited 2014 Apr 26]. p. 353-561. Available from: http://link.springer. com/chapter/10.1007/978-3-662-07856-3 19.

2. Iwamoto Y. Diagnosis and treatment of Ewing's sarcoma. Jpn J Clin Oncol. 2007;37(2):79-89.

3. Yousefi Z, Sharifhi N, Hasanzadeh M, Mottaghi M, Bolandy S. Peripheral primitive neuroectodermal tumor of the pelvis. Iran J Med Sci. 2014;39(1):71-74.

4. Burgers JM, Oldenburger F, de Kraker J, van Bunningen BN, van der Eijken JW, Delemarre JF, Staalman CR, et al. Ewing's sarcoma of the pelvis: changes over 25 years in treatment and results. Eur J Cancer. 1997;33(14):23602367.

5. Zahm SH, Fraumeni JF, Jr. The epidemiology of soft tissue sarcoma. Semin Oncol. 1997;24(5):504-514.

6. Society NYP. Proceedings of the New York Pathological Society. 1918:110.

7. Brinkhuis M, Wijnaendts LC, van der Linden JC, van Unnik AJ, Voute PA, Baak JP, Meijer CJ. Peripheral primitive neuroectodermal tumour and extra-osseous Ewing's sarcoma; a histological, immunohistochemical and DNA flow cytometric study. Virchows Arch. 1995;425(6):611616.

8. Cavazzana AO, Ninfo V, Roberts J, Triche TJ. Peripheral neuroepithelioma: a light microscopic, immunocytochemical, and ultrastructural study. Mod Pathol. 1992;5(1):71-78.

9. Kushner BH, Meyers PA, Gerald WL, Healey JH, La Quaglia MP, Boland P, Wollner N, et al. Very-highdose short-term chemotherapy for poor-risk peripheral primitive neuroectodermal tumors, including Ewing's sarcoma, in children and young adults. J Clin Oncol. 1995;13(11):2796-2804. 